\title{
Third International Conference of Students of Systematic Musicology (SysMus10): A Conference Report
}

\author{
NINA JUKIĆ \\ Department of Musicology, University of Zagreb, Croatia \\ MATS KÜSSNER \\ Department of Music, King's College London, UK \\ LI-CHING WANG \\ Graduate Institue of Musicology, National Taiwan University, Taiwan
}

\begin{abstract}
SysMus10, the third International Conference of Students of Systematic Musicology, was held at the University of Cambridge, UK, in September 2010. The conference was organised by PhD students at the Centre for Music and Science in the University's Faculty of Music. SysMus10 brought together around 40 advanced students working in the field of systematic musicology representing 14 nationalities. The presentations primarily focused on the students' ongoing research for their PhDs or Masters' degrees. The conference included the presentation and publication of 25 peerreviewed papers and posters, keynotes from top researchers in the field (Eric Clarke, Nicholas Cook, and Petri Toiviainen), a workshop and several social activities. Although the conference revealed that the concept of "systematic musicology" is still not known much outside the German-speaking research community, it served as an excellent exchange platform for students doing music research in various disciplines. SysMus10 successfully continued the strong work of the first two SysMus conferences (SysMus08, held in Graz, Austria, and SysMus09, held in Ghent, Belgium), and no doubt next year's conference, SysMus11 (to be held in Cologne, Germany), will be just as enlightening and inspiring for young musicologists and students of other fields alike.
\end{abstract}

Submitted 2010 November 26; accepted 2010 December 7.

KEYWORDS: systematic musicology, SysMus, student conference, science, humanities

\section{AS IT HAPPENED}

FOLLOWING the two previous SysMus conferences held in Graz, Austria (2008), and Ghent, Belgium (2009), the third International Conference of Students of Systematic Musicology (SysMus10) took place at the Faculty of Music, University of Cambridge, from September 13th-15th, 2010. The conference was organised by Michelle Phillips, Sarah Knight, Tal-Chen Rabinowitch and Guy Hayward, under the supervision of Ian Cross from the Centre for Music and Science. SysMus10 was generously supported by SEMPRE (http://www.sempre.org.uk/index.html), the Faculty of Music (University of Cambridge), Trinity College (Cambridge), Wolfson College (Cambridge), the Music and Letters Trust and the Society for Music Analysis (SMA). The conference series' aim to bring together young reseachers of various backgrounds was reflected in the SysMus10 programme, which included a wide scope of topics in both paper and poster sessions. Sixteen double-blind peer-reviewed papers were divided into five sessions with the following topics: Music and Culture; Expression and Emotion; Music, Language and Timing; Performance and Practice; and Music Parameter and Multimodality. The poster session comprised nine contributions from diverse research fields. SysMus10 participants (as well as the international student peerreviewers) came from numerous disciplines such as psychology, sociology, linguistics, computer science, education, arts and musicology, thus allowing viewpoints from different research fields to be exchanged and integrated. 
In his brief welcome note, Ian Cross expressed his delight to see the third SysMus conference taking place at a UK university, even though the term "systematic musicology" is neither common in the UK nor the US. He then postulated that all research should be systematic, and that the suggestion if only implicit, that there was a subdiscipline such as "unsystematic musicology" ought to be avoided. To the best of our knowledge, SysMus10 was the first academic event in the UK with the term "systematic musicology" in its title, thus marking a historic event.

\section{The keynote speakers}

After his thought-provoking remarks, Cross made way for the first of three keynote speakers, Petri Toiviainen from the University of Jyväskylä in Finland, whose paper was entitled "Music and Movement". Based on theoretical accounts of embodied cognition and the common coding theory, Toiviainen presented work that covered topics such as music-induced movement, social interaction, music and dance, and musicians' interaction and communication [1]. The method probably used most frequently to investigate movements nowadays (and certainly most enjoyable, considering the presentation of results) is called "motion capture" or simply "mocap" [2]. Toiviainen showed some cleverly designed studies from his laboratory investigating the influences of musical features, personality traits and mood on people's movements to music, and further explained that "mocap" can also be applied to examine the kinematics of musical interaction, e.g., in collective entrainment. Evidently, from his point of view, music is a spatiotemporal phenomenon.

It is highly likely that the second keynote speaker, Eric Clarke from the University of Oxford, UK, shared this view, given that, independently of Toiviainen's presentation, his contribution was entitled "Music, Motion and Subjectivity: A Workshop on Systematic Approaches" [3]. The first part of Clarke's contribution consisted of a lecture in which he started off with a philosophical approach linking music and motion to Greek philosophy and illustrated eclectic ways to deal with this topic: through metaphor, tonal space, vestibular stimulation and ordinary perception. Thus, neatly approaching music and motion/movement [4] from a different perspective than Toiviainen, Clarke discussed how sound and music are capable of conveying feelings of motion in the listener. He supported his arguments with empirical findings from the vast realm of (music) perception and action research and also drew on some musicological writings on subjectivity [5]. Equipped with such a variety of approaches, the SysMus10 participants were then asked to debate in groups how one could tackle subjective motion in music from five different perspectives, namely experimental; empirical, but not experimental; neuroscientific; analytical (focused on musical material); and discourse-based (critical reception, literature etc.).

The third keynote speaker, Nicholas Cook from the University of Cambridge, UK, whose talk was entitled "Bridging Systematic and Cultural Musicology within Performance Studies", combined at least two of those approaches very elegantly to strengthen his argument. During his presentation, Cook repeatedly made use of videos of concert pianists to emphasize the visual aspect of the perception of a piano performance. In addition, he presented results which emerged from his work at CHARM [6] (Centre for the History and Analysis of Recorded Music), demonstrating how pianists throughout the 20th century, and specifically within the Russian tradition, vary tempo and dynamics in order to shape phrases. Applying these quantifiable tools, Cook was able, for instance, to deconstruct the myth of the decline or homogenization of (piano) performances after the Second World War as put forward by Adorno. To end with, he impressively connected phrase-arching in music with modern architecture and car models (!) of that time.

\section{The student activities}

Having carried out all the necessary work to progress from initial ideas to the appearance of their papers in the SysMus proceedings [7], the student presenters then also gave professional talks at a high academic level. The inspiring and consistently enthusiastic discussions that followed encouraged brainstorming, gave rise to fresh thoughts and enlightened future perspectives.

Not only the formal discussions, but also the well-planned leisure time activities facilitated the flow of ideas between participants - in particular punting on the River Cam and traditional Scottish folk dancing (Ceildhe) at Wolfson College. The former gave the students the chance to taste the nature of Cambridge; the latter enabled the experience of British culture and embodiment of music. Although 
presumably also abetted by the fact that SysMus10 participants stayed together from breakfast through to late evening events for three days, the cooperation and interaction through these activities forged friendships as well as professional relationships. One remarkable observation was that many students who had previously attended a SysMus conference also participated in SysMus10, and recommended the conference to their friends and colleagues. Consequently, besides the academic exchange, SysMus 10 offered the opportunity to meet old friends.

The organisers of the previous SysMus conferences, Manuela Marin and Luiz Naveda, both attended SysMus10 and expressed their positive feelings and wishes in the closing session. They also presented the new SysMus conference series website, which provides information about SysMus and systematic musicology in general: https://sites.google.com/site/sysmusconference/. On behalf of the SysMus Council, they announced that the fourth International Conference of Students of Systematic Musicology will be held in Cologne, Germany, from October 5th-7th in 2011. Julia Wewers will continue the mission of the SysMus10 team under the supervision of Uwe Seifert, Head of the Musicology Department at the University of Cologne.

In summary, SysMus10 provided an excellent exchange platform for international students pursuing music research in various disciplines and warmly encouraged the sharing of experiences and findings about music. All contributions were intended to enhance the understanding of music as a multifaceted phenomenon, but crucially, the underlying questions were approached through different channels. This knowledge, grounded in different disciplines, then converged into one common "SysMus space", thus enabling interdisciplinary dialogues and different responses to general questions about music.

\section{SOME REFLECTIONS ON THE CONCEPT OF THE SYSMUS SERIES}

The term "systematic musicology" originates from Guido Adler's (1885) article "The Scope, Method, and Aim of Musicology", where he famously divided musicology into a "historical" and a "systematic" branch. His original division regarded ethnomusicology as being part of systematic musicology. Only later ethnomusicology developed into a separate branch of musicology, leading to the tripartite concept of musicology common in mainland Europe in our days. Adler's distinction has had, of course, a larger influence on music research developments in the German-speaking countries, and the Middle-European cultural circle in general, than in English-speaking and non-European countries (Leman, 2008), which has led to different understandings of the term "systematic musicology" amongst contemporary music scholars of varying educational and cultural backgrounds. Is systematic musicology as a discipline primarily defined by its methods or by the questions that it asks? These differing approaches to characterising a discipline compound the problem of giving systematic musicology a clear definition. Further cause for confusion regarding the term "systematic musicology" is the division of musicology into "historical" and "systematic" - perspectives that were probably more relevant at the end of the $19^{\text {th }}$ century when specialization and differentiation of research disciplines were promoted, and systematic musicology was considered a complement to historical musicology, rather than a discipline in its own right. However, in today's context, when new fields such as the neurosciences of music and music computing are flourishing, when many researchers have multidisciplinary backgrounds, when humanities have been influenced by post-structuralist and post-modern thought and when the world has witnessed globalisation and the overall technological progress, it might seem more appropriate to throw away the 20th century division of musicology into the three branches and see musicology rather as "any research on any music". This, of course, implies that different questions about music demand different methods to answer them - and those methods can come from various disciplines, whether it be the natural sciences or humanities. The need for interdisciplinarity and bringing together researchers from different subfields of contemporary musicology in order to answer specific musicological questions is thus stressed (these were also the conclusions of the lively post-conference discussion at SysMus08). The term "systematic musicology", however, truly only seems valid in opposition to historical musicology in the original Adlerian sense and when defining a discipline by the questions it tries to answer. In contrast to historical musicology, systematic musicology is less concerned with specific manifestations of music.

Bearing in mind what has been said above, it is interesting to observe certain differences in approaches to the concept of "systematic musicology" at the SysMus conferences held so far. The traditional Adlerian definition of systematic musicology, as well the more current definitions proposed by de la Motte-Haber (2004-2010), Kendall and Savage (2005) and Parncutt (2007), concern both natural sciences [8] and humanities. Thus, if a conference has "systematic musicology" in its title, it would be 
expected that both the scientific and the humanities sides of musicology would be equally represented in the students' papers and posters. Unfortunately, this was not the case at SysMus10 - the humanities side of musicology was noticeably underrepresented, which was also the case at SysMus09 (only SysMus08 offered a more or less balanced ratio between empirical/quantified/data-based papers and papers relying on methods from the humanities).

If we agree that "systematic" and "empirical/quantified/data-based" are not synonyms (as Clarke stressed in his talk), then there should be more contributions from the humanities at the upcoming SysMus conferences (especially in light of the difficult situation humanities have found themselves in recently funding problems, the closing of departments, etc.). Perhaps the idea behind the SysMus conference series should be the one of bringing together student researchers from both science and humanities parts of musicology. SysMus could be a platform for humanities students to gain insight into the world of quantified-data research; just as empirical research students could gain insight into cultural/historical/philosophical points of view on musical issues. Both groups of students could learn something new and get feedback from their colleagues. If the scientific and humanities sides are not well balanced, there can be no proper feedback to the papers from the underrepresented side, nor can there be true insight into different methods of music research.

Possible solutions to all of these issues will hopefully be found shortly at SysMus11 in Cologne. Since the point of meeting at conferences is not only about giving answers, but also about raising questions, it can truly be said that the SysMus series project has been extremely successful so far, and we can strongly recommend to all young music researchers to apply for the next conference. All information on the past and future SysMus conferences is to be found on the series' official website: https://sites.google.com/site/sysmusconference/home.

\section{NOTES}

[1] For instance, SysMus10 participants learned that more and more neuroscientific methods are used to study beat perception and its underlying activations of the motor areas of the brain, and that there is also research into the vestibular system and how it reacts when one "nods to the beat".

[2] By placing sensors on different parts of the body, motion capture enables researchers to quantify and track people's movements meticulously.

[3] It is worth mentioning here that Clarke stressed the fact that "systematic" does not necessarily mean "quantified."

[4] During the final discussion, there was agreement amongst SysMus10 participants that there is a fine distinction between these two terms, linking movement to discrete, real, actual and physical processes and motion to continuous, abstract and virtual ones. It was also noted that this distinction may be specific to English and not present in all languages.

[5] One of Clarke's arguments was that motion implies the presence of a body/subject.

[6] CHARM was an AHRC-funded research project running from 2004-09 which is now followed by the Centre for Musical Performance as Creative Practice (CMPCP).

[7] The SysMus10 Proceedings can be downloaded from the following website: https://sites.google.com/site/sysmusconference/sysmus-proceedings.

[8] Many people whose native language is not English understand the meaning of the word "science" in very close relation to its Latin origin (scientia or knowledge), while in English the word has come to denote primarily the so-called "natural sciences" (in German - Naturwissenschaft). In other words, in many languages "science" simply means any systematic research, and is not opposed to humanities (in German Geisteswissenschaft), as is the case in English. 


\section{REFERENCES}

Adler, G. (1885). Umfang, Methode und Ziel der Musikwissenschaft. Vierteljahresschrift für Musikwissenschaft, Vol. 1, pp. 5-20.

Kendall, R. A. \& Savage, R. (Eds.) (2005). Perspectives in systematic musicology: Selected reports in ethnomusicology (Vol.12). University of California: Department of Ethnomusicology.

Leman, M. (2008). Systematic musicology at the crossroads of music research. In: Schneider A. (Ed.), Systematic and Comparative Musicology: Concepts, Methods, Findings. Hamburger Jahrbuch für Musikwissenschaft 24, Frankfurt am Main: Peter Lang, pp. 89-115.

Motte-Haber, H. de la (2004-2010). Handbuch der Systematischen Musikwissenschaft in 6 Bänden. Laaber: Laaber Verlag.

Parncutt, R. (2007). Systematic musicology and the history and future of western musical scholarship. Journal of Interdisciplinary Music Studies, Vol. 1, No. 1, pp. 1-32. 